\title{
Sensate composite calcaneal flap in leg amputation: a full terminal weight-bearing surface-experience in eight adult patients
}

\author{
Bruno Livani · Gabriel Castro $\cdot$ Jose Roberto Tonelli Filho • \\ Tâmara Ramos Morgatho • Mauricio Leal Dias Mongon • \\ William Dias Belangero · Michael Davitt · Jose André Carvalho
}

Received: 27 February 2011/ Accepted: 11 July 2011/Published online: 26 July 2011

(C) The Author(s) 2011. This article is published with open access at Springerlink.com

\begin{abstract}
Despite modern reconstruction techniques and replantation, the preservation of a severely traumatised limb, or even a limb affected by a congenital malformation, usually gives poorer functional results compared with amputation and prosthetisation. The aim of this study was to describe a hind foot (including the calcaneum and fat pad) sensate flap with a surface that allows full terminal weight bearing in transtibial amputations in adults. Between June 2007 and September 2008, eight patients underwent leg amputations with a sensate composite calcaneal flap reconstruction of the stump. Patients consisted of four men and four women with a mean age of 46.5 (26-66) years. All amputations were unilateral. The mean follow-up was 28.3 (25-42) months. There were no complications. Calcaneum tibial fusion was observed in all patients in a mean time of 3.5 (3-4) months. A below-knee prosthesis was adapted at 16 weeks postoperatively in all cases, and no need for stump revision occurred in this series during the entire follow-up period. A transtibial amputation covered with a sensate plantar flap preserving the calcaneum was proposed. In theory, the anatomic structures spared in this technique provide a strong, full, weight-bearing terminal surface of the stump that will last a lifetime.
\end{abstract}

Keywords Heel $\cdot$ Leg $\cdot$ Extremities

\footnotetext{
B. Livani $(\bowtie)$

Rua Ana Zorzetto Favaro 18, Residencial Paineiras,

Bairro Betel, São Paulo 13140-000, Brazil

e-mail: brunolivani@hotmail.com

G. Castro - J. R. T. Filho - T. R. Morgatho .

M. L. D. Mongon - W. D. Belangero - M. Davitt .

J. A. Carvalho

Hospital Madre Theodora, Campinas, SP, Brazil
}

\section{Introduction}

Despite modern reconstruction techniques and replantation, the preservation of a severely traumatised limb, or even a limb affected by a congenital malformation, usually gives poorer functional results compared to amputation and prosthetisation [1].

Of the possible levels of amputation, transtibial amputations result in functionally excellent outcomes. However, in contrast to hind foot amputations, such as Syme and especially Boyd and Pirogoff amputations, acute or late complications related to the amputated stump are frequent with the various described methods [2].

In 1949, Ertl described a surgical technique that restored the interosseous pressure through canal obliteration and expanded the area of terminal support by forming a bony bridge between the fibula and distal tibia, similar to Boyd amputation, but without the calcaneum and fat pad [3-5].

The aim of this study was to report the results of a hind foot (including the calcaneum and fat pad) sensate flap with a surface that allows full terminal weight bearing in transtibial amputations in adults.

\section{Patients and methods}

Patients

Between June 2007 and September 2008, eight patients underwent leg amputations with a sensate composite calcaneal flap reconstruction of the stump Table 1.

The inclusion criteria were adult patients who had indications for midshaft or distal tibial leg amputations. The exclusion criteria were patients with calcaneum disease such as osteomyelitis, heel pad ulcers, absence of a 
Table 1 Preoperative data

\begin{tabular}{|c|c|c|c|c|c|c|}
\hline Patient & Sex & Age, years & Aetiology & Comorbidities & Fixation & Flap \\
\hline 1 & Male & 66 & Chronic distal tibial osteomyelitis & Schizophrenia diabetes & Two Steinmann pins & Pedicled \\
\hline 2 & Female & 43 & Infected distal tibial pseudarthrosis & None & Two Steinmann pins & Pedicled \\
\hline 3 & Female & 38 & Infected distal tibial pseudarthrosis & None & Two Steinmann pins & Pedicled \\
\hline 4 & Male & 49 & Chronic distal tibial osteomyelitis & None & Two Steinmann pins & Pedicled \\
\hline 5 & Male & 26 & Chronic tibio-talar infection osteomyelitis & Vascular injured limb & Two Steinmann pins & Pedicled \\
\hline 6 & Male & 49 & Atrophic distal tibial pseudarthrosis & None & Two Steinmann pins & Pedicled \\
\hline 7 & Female & 43 & Infected midshaft leg pseudarthrosis & None & Ilizarov external fixator & Free flap \\
\hline 8 & Female & 58 & Chronic distal tibial osteomyelitis & None & Two Steinmann pins & Pedicled \\
\hline
\end{tabular}

posterior tibialis pulse, a nonsensible plantar foot sole, especially in the heel fat pad region, and oncologic indications for leg amputation.

Patients consisted of four men and four women with a mean age of 46.5 (26-66) years. All amputations were unilateral.

The mean follow-up was 28.3 (25-42) months.

\section{Operative technique}

The sensate composite calcaneal flap should be fixed as far distally as possible. The surgical procedure began with a medial longitudinal ankle incision to identify and isolate the posterior tibial neurovascular bundle, which was dissected proximally (until the tibial osteotomy level) and distally, including the calcaneum, heel fat pad, and as much foot sole as possible. A midfoot disarticulation at Chopart's joint was then performed as described by Boyd, with talectomy and calcaneus articular cartilage surface removal.

A midshaft tibial osteotomy was performed, and the distal flap was still perfused and innervated by the intact posterior tibial neurovascular bundle Fig. 1. To provide a tibial calcaneum fusion, the heel was fixed to the remaining tibial shaft with two smooth Steinmann pins. In the last case, we performed the fusion with an Ilizarov external fixator Fig. 2.

The neurovascular bundle remained intact in all cases except one, in which the neurovascular bundle was too redundant due to a shortening longer than $20 \mathrm{~cm}$; we decided to resect a segment of the posterior tibial artery and veins with a termino-terminal microanastomosis, leaving the nerve intact, although with redundant nerve looping.

The patients were clinically and radiographically evaluated monthly. The stitches were removed after 3 or 4 weeks postoperatively.

A below-knee cast was used in the first seven cases until bone healing was observed both clinically and radiographically. The cast and Steinmann pins were then removed, and the patients started a rehabilitation and prosthetisation programme.

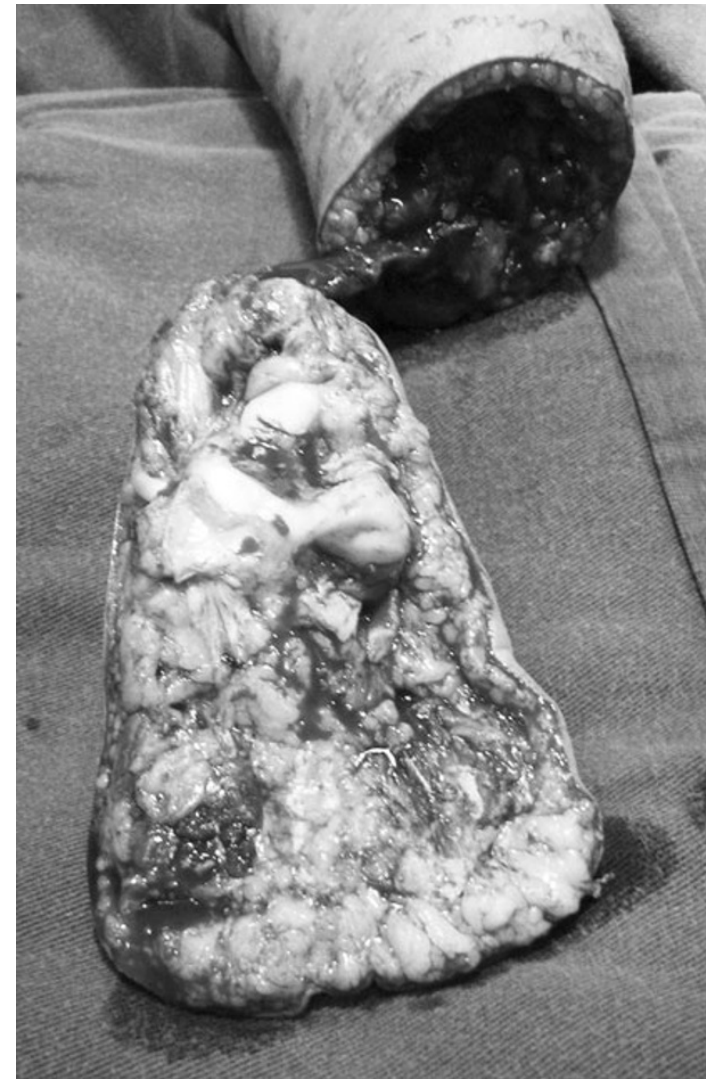

Fig. 1 Intraoperative picture showing a pedicled sensate composite calcaneal flap

\section{Results}

The intraoperative procedure had no intercurrences. The wound had no infection, and skin incisions healed without complications in all cases.

The stumps were painless and able to bear total terminal weight at 16 weeks in all patients. Calcaneum tibial fusion was observed in all patients in a mean time of 3.5 (3-4) months Table 2. Fusion was considered when the patients were completely pain free, no motion between the fragments could be clinically demonstrated, and the radiographic exam 


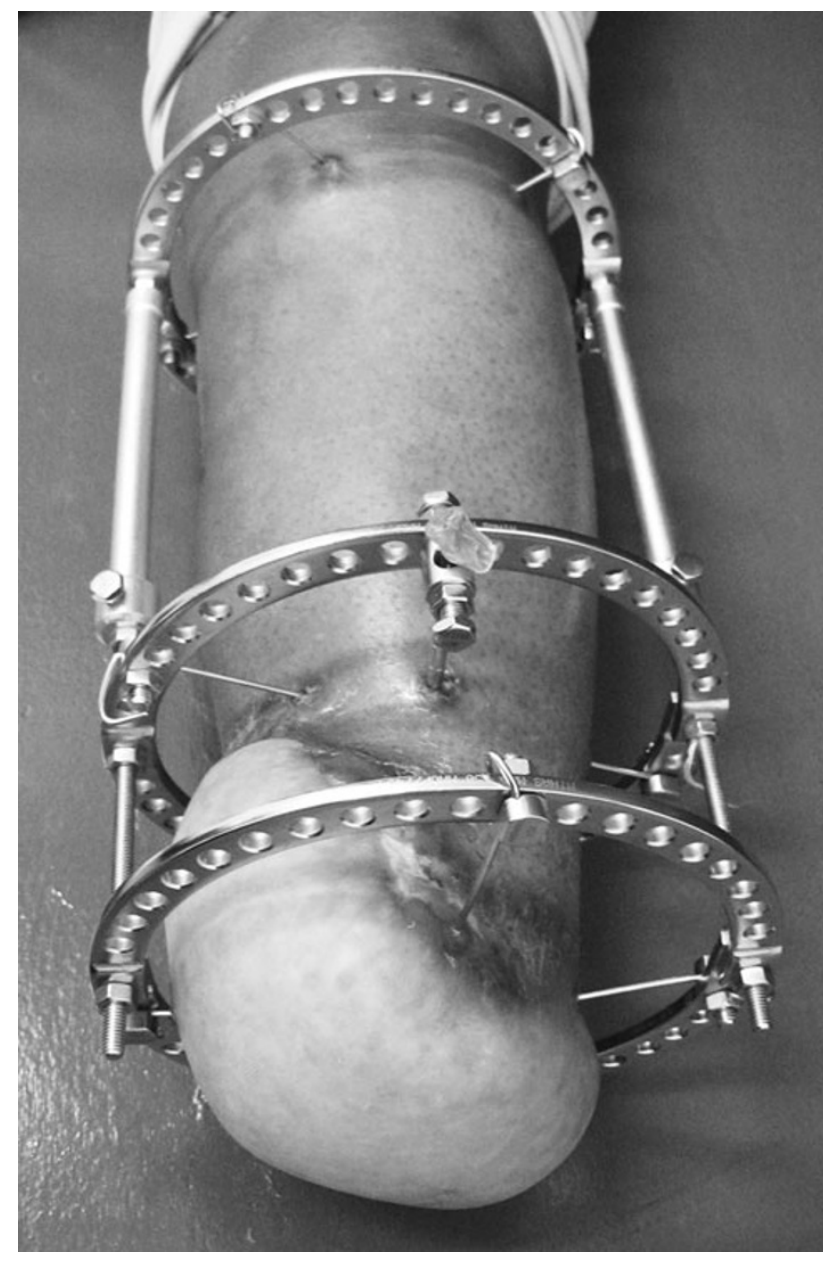

Fig. 2 Picture at 3 months postoperative follow-up showing the flap fixed and compressed by an Ilizarov external fixator

Table 2 Postoperative data

\begin{tabular}{llll}
\hline Patient & $\begin{array}{l}\text { Follow-up } \\
\text { (months) }\end{array}$ & $\begin{array}{l}\text { Healing (fusion) } \\
\text { time (months) }\end{array}$ & Complications \\
\hline 1 & 42 & 03 & None \\
2 & 35 & 03 & None \\
3 & 33 & 04 & None \\
4 & 31 & 03 & None \\
5 & 30 & 04 & None \\
6 & 29 & 04 & None \\
7 & 29 & 04 & None \\
8 & 25 & 03 & None \\
\hline
\end{tabular}

showed the presence of bony trabeculae continuity through the main fragments (distal tibia and calcaneum) Fig. 3.

A below-knee prosthesis was adapted at 16 weeks postoperatively in all cases, and no need for stump revision occurred in this series during the entire follow-up period.

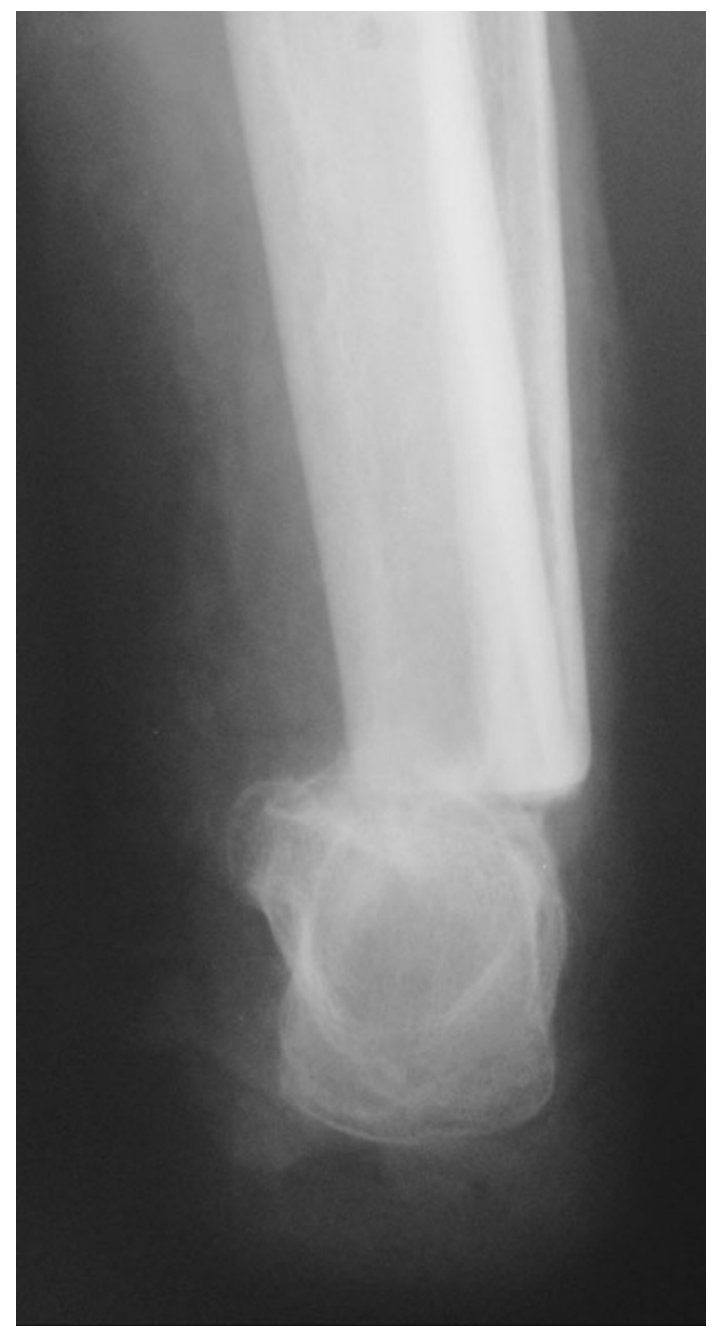

Fig. 3 Picture at 30 months postoperative follow-up showing the calcaneal flap fused to distal tibia

\section{Discussion}

Generally, the quality of life of a lower-limb amputee with a functional stump is similar to that of an average person [6]. Consequently, a technically well-constructed stump must be the objective of the orthopaedic surgeon. Unfortunately, many amputations are delegated to less experienced orthopaedic surgeons or even to medical residents, which can lead to poor functional results, chronic stump pain and prosthetisation difficulties. In the classical transtibial amputation, the fibula is osteotomised approximately $1 \mathrm{~cm}$ shorter than the tibia, avoiding an excessive concentration of pressure at this location during the gait after prosthetisation. The common complications with this technique may require stump revisions and result in high late-morbidity rates [7].

The initial amputation stump may be funnel-shaped, which can cause pain and difficulty adapting to a prosthesis. The distal narrowing of the stump is caused by 
posteromedial migration of the fibula, which tends to medialise with weight bearing because it lacks a distal contention (i.e. the syndesmosis is lost). Given that pressure equals force divided by area $(P=\mathrm{F} / \mathrm{A})$, a smaller stump area experiences greater pressure and is thus more likely to develop ulcers, late infection and pain. Conversely, a larger area of support allows for a wider distribution of pressure, which reduces the likelihood of pain and increases the weight-bearing ability of the terminal stump. This is particularly important in meeting the greater functional demands in patients such as young people, athletes, members of the military and professionals who exert more physical effort [8]. In addition, a delay in or absence of the use of a stump can lead to progressive osseous demineralisation (nonuse osteoporosis), which is generally painful; the pain further reduces stump use, leading to a vicious circle that incapacitates the patient. Often, socioeconomic difficulties result, and an associated depression develops: the sooner the patient returns to a daily routine, the greater the chances are for socioeconomic readaptation [9].

Ankle disarticulations provide a durable end-bearing stump. In 1843, Syme described an amputation consisting of a bone section at the distal tibia and fibula $6 \mathrm{~mm}$ proximal to the periphery of the ankle joint. The tough, durable skin and fat pad of the heel flap provide normal weight-bearing skin. The most common cause of an unsatisfactory Syme stump is posterior migration of the heel pad [10, 11].

The Boyd amputation produces an excellent end-bearing stump around the ankle and eliminates the problem of posterior migration of the heel pad in addition to providing a stable stump with an intact proprioceptive sensation. This procedure involves talectomy, a forward shift of the calcaneus and calcaneotibial fusion [10, 11].

When classical transtibial amputation is performed, skin complications such as wound breakdown and development of ulcers, and callosities over a disarticulated stump may occur because of the concentration of pressure on the small weight-bearing stump. These complications often prolong the patient's hospital stay and prevent early aggressive rehabilitation with a prosthesis. To overcome this problem, a greater weight-bearing area and sufficient soft tissue coverage are desirable [4].

In 1949, Ertl described an amputation technique using a bony bridge that in theory dealt with all of the inconveniences of the conventional transtibial amputation previously mentioned. This procedure closes the medullary canal with cortical bone, which restores the intraosseous pressure, blood flow and vascularisation. The resulting bony bridge increases the terminal area of the stump, makes it more stable and avoids the posteromedial migration of the fíbula and the consequent funnelling. A larger base distributes the weight over a wider area, reducing the pressure [4-7].

Jupiter et al. and Ghali et al. both reported very good results using a fillet of a foot flaps, in traumatic amputations of the leg when the foot was intact. In both reports, the authors performed a pedicled flap of the foot sole and the heel pad without the calcaneum bone [12, 13].

In 2000, Stiebel et al. described a sensate calcaneal flap in traumatic leg amputations. The authors performed a pedicled calcaneal flap with the calcaneum turned on at a $90^{\circ}$ in its longitudinal axis and fixed to the tibia as in Pirogoff amputation [14]. They fixed the calcaneum to the tibia in an elegant way using two crossed lag screws [14].

In 2002, Weber described a new technique for capping the distal tibia with a neurovascular calcaneus pedicle flap in congenital pseudarthrosis of the tibia in children. The technique was very similar to the technique described by Stiebel et al's. In that article, the author had not performed any of these procedures in adults, but recommended segmental resection of the vascular bundle and terminal anstomosis to avoid arterial occlusion because adults lack the vessel elasticity to allow loop arrangement and vein thrombosis [15].

We performed a pedicled composite calcaneal flap in all patients but one. In all cases, the calcaneum was fixed to the tibia similar to a Boyd amputation. In this series, we decided to proceed with a segmental vascular bundle resection with a termino-terminal microanastomosis reconstruction performing a free flap in only one patient. In this patient, the vascular bundle remained too redundant because the shortening was longer than $20 \mathrm{~cm}$; although the flap was still perfused, we were concerned about a later perfusion compromise due to arterial or venous occlusion. To facilitate the clinical postoperative flap observation, we decided to use an Ilizarov external fixator instead of Steinmann pins and cast. The healing time was almost identical to that of the other patients, but we believed that it was more comfortable than using a cast for 3-5 months. Therefore, we intend to use the Ilizarov fixator more often in future cases, even in pedicled flaps. Another option would be to provide calcaneum fixation to the tibia using lag screws as proposed by Stiebel et al's. [14].

The procedures had no complications, even with some vascular bundle redundancy and looping. All calcaneal flaps consolidated between 3 and 5 months (average, 3.5 months).

We did not request routine preoperative angiographic studies in all patients. We only performed this examination in Patient 5, who had a vascular limb reconstruction after a knee dislocation and popliteal artery injury. This patient developed compartment syndrome and was treated with leg fasciotomy. Although the vascular bypass was able to maintain the limb, big toe necrosis and amputation, foot 


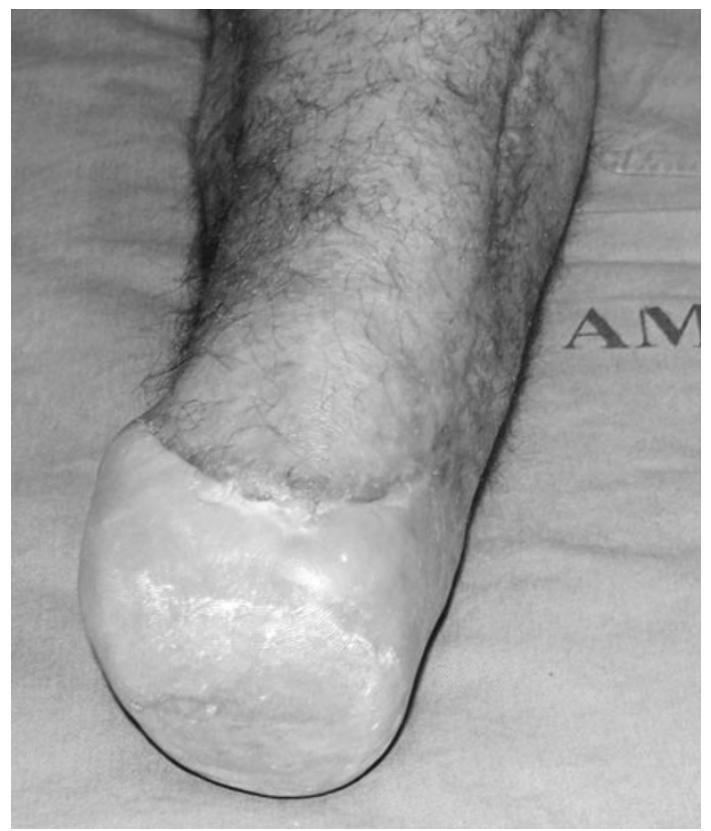

Fig. 4 Picture showing the clinical aspect of the stump at 30 months postoperative follow-up

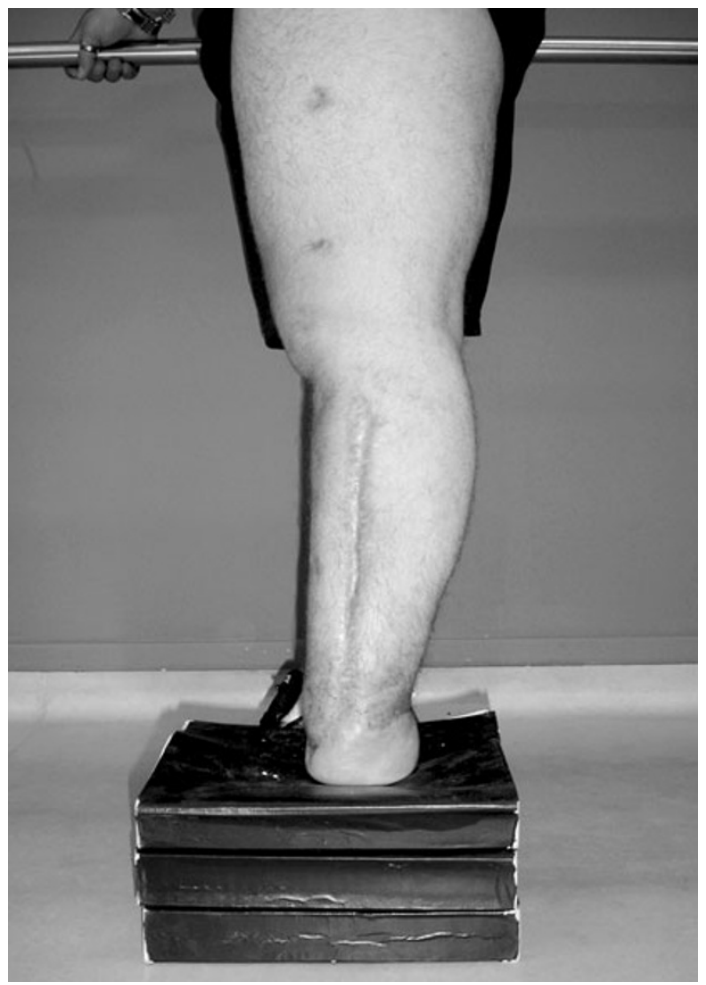

Fig. 5 Picture showing the patient able to support a painless full terminal weight bearing at 30 months postoperative follow-up

deformity with tíbio-talar chronic infection occurred. Because the angiographic examination revealed that the hind foot was perfused, the patient elected to undergo the procedure described above.
The flap described provides proprioceptive feedback with the best bone and skin to support weight bearing. Another advantage is the possibility of using the same prosthesis commonly used in Boyd or Syme amputations due to a longer arm leverage that also allows full terminal weight bearing. The suspension system used to provide prosthesis fixation was the calcaneum itself, as the bulky distal surface, which looks like a 'mallet', promotes a stronger construction that avoids the usual supracondilean suspension system used in the most common KBM prosthesis Figs. 4 and 5. The obvious disadvantage of this procedure is that this amputation technique is only possible when the patient has a healthy calcaneum and heel pad.

\section{Conclusion}

In the current study, a transtibial amputation covered with a sensate plantar flap preserving the calcaneum was proposed. In theory, the anatomic structures spared in this technique provided a strong, full, weight-bearing terminal surface of the stump that will last a lifetime.

Acknowledgments Funding: FAPESP (São Paulo State Research Foundation), São Paulo, Brazil: 2009/02089-3. Profs.: Rames Mattar Jr, Marcelo Rosa de Rezende and Gustavo Bispo dos Santos, IOTFMUSP, São Paulo, Brazil. Prof. Lourenço Sbragia Neto, FMRPUSP, Ribeirão Preto, SP, Brazil.

Conflict of interest The authors report no conflict of interest.

Open Access This article is distributed under the terms of the Creative Commons Attribution License which permits any use, distribution and reproduction in any medium, provided the original author(s) and source are credited.

\section{References}

1. Tekin L, Safaz Y, Göktepe AS, Yazýcýodlu K (2009) Comparison of quality of life and functionality in patients with traumatic unilateral below knee amputation and salvage surgery. Prosthet Orthot Int 33(1):17-24

2. Ebrahimzadeh MH, Hariri S (2009) Long-term outcomes of unilateral transtibial amputations. Mil Med 174(6):593-597

3. Deffer PA, Moll JH, LaNoue AM (1971) The Ertl osteoplastic below-knee amputation. J Bone Joint Surg Am 53:1028

4. Pinzur MS, Beck J, Himes R, Callaci J (2008) Distal tibiofibular bone-bridging in transtibial amputation. J Bone Joint Surg Am 90(12):2682-2687

5. Gwinn DE, Keeling J, Froehner JW, McGuigan FX, Andersen R (2008) Perioperative differences between bone bridging and nonbone bridging transtibial amputations for wartime lower extremity trauma. Foot Ankle Int 29(8):787-793

6. Pinzur MS, Pinto MA, Saltzman M, Batista F, Gottschalk F, Juknelis D (2006) Health-related quality of life in patients with transtibial amputation and reconstruction with bone bridging of the distal tibia and fibula. Foot Ankle Int 27:907-912 
7. Pinto MA, Harris WW (2004) Fibular segment bone bridging in trans-tibial amputation. Prosthet Orthot Int 28:220-224

8. Legro MW, Reiber GD, Smith DG, del Aguila M, Larsen J, Boone D (1998) Prosthesis evaluation questionnaire for persons with lower limb amputations: assessing prosthesis-related quality of life. Arch Phys Med Rehabil 79:931-938

9. Harness N, Pinzur MS (2001) Health related quality of life in patients with dysvascular transtibial amputation. Clin Orthop Relat Res 383:204-207

10. Grady JF, Winters CL (2000) The Boyd amputation as a treatment for osteomyelitis of the foot. J Am Podiatr Med Assoc 90(5):234-239

11. Pinzur MS, Gottschalk FA, Pinto MA, Smith DG (2007) Controversies in lower-extremity amputation. J Bone Joint Surg Am 89:1118-1127
12. Jupiter JB, Tsai TM, Kleinert HE (1982) Salvage replantation of lower limb amputations. Plast Reconstr Surg 69(1):1-8

13. Ghali S, Harris PA, Khan U, Pearse M, Nanchahal J (2005) Leg length preservation with pedicled fillet of foot flaps after traumatic amputations. Plast Reconstr Surg 115(2):498-505

14. Stiebel M, Lee C, Fontes R (2000) Calcaneal fillet of sole flap: durable coverage of the traumatic amputation stump. J Trauma 49(5):960-963

15. Weber M (2002) Neurovascular calcaneo-cutaneus pedicle graft for stump capping in congenital pseudarthrosis of the tibia: preliminary report of a new technique. J Pediatr Orthop B 11:47-52 